\title{
Papillary thyroid microcarcinoma: time to shift from surgery $Q$ to active surveillance?
}

\author{
Sophie Leboulleux, R Michael Tuttle, Furio Pacini, Martin Schlumberger
}

The incidence of differentiated thyroid cancer is increasing greatly in high-income countries. Roughly $50 \%$ of this increase is attributable to the identification of intrathyroidal papillary thyroid microcarcinomas. Since mortality associated with these tumours remains low and stable, the increasing diagnosis has led to concerns about overdiagnosis and overtreatment. Management of papillary thyroid microcarcinomas should take into account the reported absence of mortality when diagnosed in the absence of lymph node metastases and distant metastases, as shown even in recent studies promoting active surveillance; a low recurrence rate of 1-5\%; and the risk of permanent complications from surgery that cannot be decreased to less than 1-3\%, even in high-volume tertiary care centres with experienced surgeons. On the basis of these data, active surveillance with curative intent, in which active treatment is delayed until the cancer shows signs of significant progression to avoid side-effects of treatment, should be considered in properly selected patients.

\section{Introduction}

Papillary thyroid microcarcinomas are small thyroid cancers measuring $1 \mathrm{~cm}$ or less. Most of these tumours are not palpable and are identified either through pathology examination of thyroid glands removed for benign conditions or from imaging procedures such as neck ultrasonography or CT scan for another cause. Much less commonly, papillary thyroid microcarcinoma presents with palpable neck node metastases or distant metastases, leading to the diagnosis of a yet unknown papillary thyroid cancer.

\section{Epidemiology}

Occult papillary thyroid microcarcinomas at autopsy

Findings from autopsy studies have shown that occult papillary thyroid microcarcinomas are present in 5-36\% of the population (table 1). ${ }^{1-11}$ In a meta-analysis of 989 autopsies from 15 studies, the prevalence was $11.5 \% .^{12}$ Occult papillary thyroid microcarcinomas are characterised by a very small size, with the tumour smaller than $1 \mathrm{~mm}$ in $33-79 \%$ of cases, and a high frequency of multifocality, which is present in $27-50 \%$ of cases (table 1). The prevalence of occult papillary thyroid microcarcinomas is higher in people older than 40 years than in younger people, but is not associated with either iodine intake or sex. The main factor affecting the prevalence of these tumours is the method used for the autopsy analysis. ' Occult papillary thyroid microcarcinomas represent a major reservoir of unknown thyroid malignancies, which we estimate to affect roughly 20 million adults in the USA and 48 million in Europe. Indeed, the prevalence of occult papillary thyroid microcarcinomas identified at autopsy is 100-1000 times higher than that of clinical cancer.

\section{Clinical papillary thyroid microcarcinomas}

There has been a substantial rise in the incidence of thyroid cancer in developed countries during the past 30 years. ${ }^{13-16}$ Roughly $50 \%$ of this increase is attributable to the identification of intrathyroidal papillary thyroid microcarcinomas, which are present in at least $10 \%$ of thyroid glands removed for benign thyroid diseases. ${ }^{15,17}$ The most common presentation of thyroid cancer is a papillary thyroid microcarcinoma, measuring less than $2 \mathrm{~mm}$ in $60 \%$ of patients, in a patient older than 45 years. ${ }^{18,19}$ At the same time, mortality from papillary thyroid carcinoma remains low and stable. ${ }^{17}$

Several changes in medical practice in high-income countries have resulted in the increased identification of papillary thyroid microcarcinomas that were previously part of a large subclinical thyroid cancer reservoir. One of these developments is the increased detection of asymptomatic incidental thyroid nodules through highly sensitive imaging procedures, such as the widespread availability of neck ultrasonography that is often used for various neck conditions such as pain, discomfort, or any kind of thyroid disease, and the availability of other imaging procedures that are done for unrelated reasons, such as carotid artery doppler studies, neck MRI for cervical discopathy, and CT scan or 18 F-fluorodeoxyglucose (18F-FDG) PET scans for non-thyroidal malignancies. Other developments that have led to increased identification of papillary thyroid microcarcinomas are the increased spatial resolution of the latest neck ultrasound machines, which can now detect nodules as small as $2 \mathrm{~mm}$; the increased ability to take biopsy samples from very small thyroid nodules by use of ultrasound-guided fine needle aspiration; and a more thorough histological scrutiny of thyroid specimens obtained at surgery for benign thyroid diseases by sectioning and examining the entire thyroid gland. ${ }^{20}$ During the past three decades, a large proportion of thyroid cancer diagnoses - up to $60 \%$ according to results from a recent study ${ }^{21}$ — can be accounted for by these diagnostic changes. The introduction of a formal screening programme with ultrasonography in South Korea resulted in an increase in diagnosis of thyroid cancer by 15 times within 10 years, with clear concern regarding its usefulness. ${ }^{22,23}$ A screening programme initiated in Fukushima following the Daiichi nuclear
Lancet Diabetes Endocrinol 2016 Published Online August 19, 2016 http://dx.doi.org/10.1016/ S2213-8587(16)30180-2

Department of Nuclear Medicine and Endocrine Oncology, Gustave Roussy and Université Paris Saclay, Villejuif, France (S Leboulleux MD, Prof M Schlumberger MD), Endocrinology Service, Memorial Sloan Kettering Cancer Center (MSKCC), New York, NY, USA (Prof R Michael Tuttle MD); and Section of Endocrinology Department of Medicine, Surgery and Neuroscience, University of Siena, Siena, Italy (Prof F Pacini MD)

Correspondence to: Dr Sophie Leboulleux, Department of Nuclear Medicine and Endocrine Oncology, Gustave Roussy, Université Paris Saclay, 114 Rue Edouard Vaillant, 94805 Villejuif, France sophie.leboulleux@ gustaveroussy.fr 


\begin{tabular}{|c|c|c|c|c|c|c|c|c|}
\hline & Country & $\mathbf{N}$ & Prevalence & Prevalence, male & Prevalence, female & Size $(\mathrm{mm})$ & Tumour size $<1 \mathrm{~mm}$ & Multifocal tumours \\
\hline Sampson et al $(1974)^{1}$ & USA & 157 & $9(5.7 \%)$ & $7 / 99$ (7.1\%) & $2 / 58(3.5 \%)$ & NA & NA & NA \\
\hline Sobrinho-Simoes et al (1979) & Portugal & 600 & $39(6 \cdot 5 \%)$ & $9 / 338(2 \cdot 7 \%)$ & $30 / 262(11 \cdot 5 \%)$ & NA & NA & $12 / 39(30 \cdot 8 \%)$ \\
\hline Bondeson et al $(1981)^{3}$ & Sweden & 500 & $39^{*}(7 \cdot 8 \%)$ & $17 / 279(6.0 \%)$ & $22 / 221(10 \cdot 0 \%)$ & NA & $N A^{*}$ & NA \\
\hline Harach et al $(1985)^{4}$ & Finland & 101 & $36(35 \cdot 6 \%)$ & $23 / 53(43 \cdot 4 \%)$ & $13 / 48(27 \cdot 1 \%)$ & NA & $40 / 52(76 \cdot 9 \%)$ & $10 / 36(27 \cdot 8 \%)$ \\
\hline Lang et al $(1988)^{5}$ & Germany & 1020 & $63(6 \cdot 2 \%)$ & $39 / 599(6.5 \%)$ & $24 / 421(5 \cdot 7 \%)$ & NA & NA & $29 / 63(46 \cdot 0 \%)$ \\
\hline Ottino et al $(1989)^{6}$ & Argentina & 100 & $11(11 \cdot 0 \%)$ & $8 / 59(13 \cdot 6 \%)$ & $3 / 41(7 \cdot 3 \%)$ & Median 2.1 & $6 / 18(33 \cdot 3 \%)$ & $3 / 11(27 \cdot 3 \%)$ \\
\hline Yamamoto et al $(1990)^{7}$ & Japan & 408 & $46(11 \cdot 3 \%)$ & $26 / 247(10 \cdot 5 \%)$ & $20 / 161(12 \cdot 4 \%)$ & Mean 1.98 & $23 / 64(35 \cdot 9 \%)$ & $14 / 46(30 \cdot 4 \%)$ \\
\hline Furmanchuk et al $(1993)^{8}$ & Belarus & 215 & $19^{*}(8 \cdot 8 \%)$ & $10 / 138(7 \cdot 2 \%)$ & 9/77 (11.7\%) & $\begin{array}{l}\text { Mean } 1.7 \\
\text { median } 1.1\end{array}$ & $13 / 28(46 \cdot 4 \%)$ & $6 / 19(31.6 \%)$ \\
\hline Martinez-Tello et al (1993) $)^{9}$ & Spain & 100 & $22(22.0 \%)$ & $16 / 66(24 \cdot 2 \%)$ & $6 / 34(17 \cdot 6 \%)$ & NA & $42 / 53(79 \cdot 2 \%)$ & $11 / 22(50.0 \%)$ \\
\hline Kovacs et al $(2005)^{10}$ & Hungary & 433 & $21(4 \cdot 7 \%)$ & NA & NA & Mean 2.45 & $9 / 21(42 \cdot 8 \%)$ & $2 / 21(9 \cdot 5 \%)$ \\
\hline Neuhold et al (2001) $)^{11}$ & Austria & 118 & $10(8.6 \%)$ & $6 / 57(10 \cdot 5 \%)$ & $4 / 61(6 \cdot 6 \%)$ & Mean 4.9 & 0 & $3 / 10(30 \cdot 0 \%)$ \\
\hline
\end{tabular}

power plant accident diagnosed thyroid cancer with an incidence 30 times higher than the national incidence in children and adolescents in both contaminated and noncontaminated regions. ${ }^{24}$ Futhermore, in high-income countries, the increase in diagnosis of papillary thyroid microcarcinomas is related to the economic status of the patients, because economic status is associated with access to medical facilities through medical health insurance. $^{19,25}$ Although financial incentives to track coding of malignancy might have contributed to the increase in diagnoses of thyroid cancer in the USA, this is not the case in France or Italy where similar increases in papillary thyroid microcarcinomas have been reported despite the absence of financial remuneration for thyroid cancer coding. ${ }^{13,26,27}$

Clinical and occult papillary thyroid microcarcinomas from autopsy series are distinct entities, as reflected by differences in their sex ratio and size. Although clinical differentiated thyroid cancer is three times more frequent in women than men, the prevalence of latent papillary thyroid microcarcinomas does not differ between men and women in autopsy studies (table 1). ${ }^{12}$ The size of papillary thyroid microcarcinomas has been shown to be larger in clinical studies than in autopsy studies. ${ }^{12}$ Cohort studies of papillary thyroid microcarcinoma include patients with both clinical and occult (found at surgery) carcinomas because there are no formal criteria to distinguish one from the other. However, the prognosis of both clinical and occult papillary thyroid microcarcinomas is excellent.

Because of the high frequency of papillary thyroid microcarcinomas in autopsy studies and the increasing incidence of clinical papillary thyroid microcarcinomas, there is concern about overdiagnosis and overtreatment of lesions that would not cause any harm if left untreated. To minimise this problem, a working group suggested a change in terminology, with use of the term indolent lesion of epithelial origin rather than cancer. ${ }^{28}$ Some radiologists suggested "turn[ing] off the ultrasound machines", ${ }^{29}$ whereas others suggested that thyroid micro-incidentalomas should be ignored rather than explored. ${ }^{30}$

\section{Natural history and prognosis \\ Mortality}

Mortality related to papillary thyroid microcarcinoma is very low, reaching up to $0.3 \%$ in clinical series that included patients with palpable lymph nodes and distant metastases at initial presentation (table 2). ${ }^{31-42}$ Furthermore, in the absence of treatment, no deaths have been attributed to thyroid cancer in patients undergoing active surveillance. ${ }^{43}$ There is no excess mortality caused by papillary thyroid microcarcinoma diagnosed without clinical lymph nodes or distant metastases.

\section{Risk of recurrence and predictive factors for recurrence}

In series with more than 250 patients, clinical recurrences occurred in 1-5\% of patients with papillary thyroid microcarcinoma, irrespective of the presence of palpable lymph nodes at initial diagnosis (table 2). ${ }^{31-41}$ When patients with palpable lymph nodes were excluded, the rate of recurrence decreased to less than $1 \% .^{33}$ In a meta-analysis, investigators reported a recurrence rate of $0.5 \%$ (four of $854)$ in patients with incidental papillary thyroid microcarcinoma (diagnosed on histological examination of thyroid specimens following thyroid surgery for noncancer diagnoses), compared with $7.9 \%$ (173 of 2669) in patients with non-incidental papillary thyroid microcarcinoma (diagnosed preoperatively); however, nonincidental papillary thyroid microcarcinoma included tumours diagnosed on imaging undertaken for nonthyroid reasons and those diagnosed because of clinical nodal metastasis, a distinct clinical entity. ${ }^{42}$

In a single-institution series of 1066 patients with papillary thyroid microcarcinoma, an increased risk of locoregional lymph node metastases at diagnosis was significantly associated with male sex, younger age ( $\leq 45$ years), multifocal lesions, extrathyroidal extension, 
and larger tumour size $(>6 \mathrm{~mm}){ }^{41}$ Furthermore, the likelihood of lateral neck lymph node involvement was associated with extrathyroidal extension, multifocal lesions, central neck lymph node involvement, and location of the tumour in the upper third of the thyroid lobe.

Microscopic extrathyroidal extension, subcapsular location of the primary tumour, and multifocal disease have been associated with a higher risk of recurrence and locoregional metastases at diagnosis in some, ${ }^{36,40,41-46}$ but not all studies. ${ }^{47-51}$ Results from other studies have suggested that the risk factors associated with recurrence might differ between younger $(<45$ years at diagnosis) and older patients. $^{52}$ In a recent meta-analysis, clinical recurrences were associated with multifocal tumours, locoregional metastases at diagnosis, and younger age, but not with sex, size of the primary tumour, or extrathyroidal extension. ${ }^{53}$ Buffet and colleagues ${ }^{40}$ proposed a scoring system to predict recurrences in papillary thyroid microcarcinoma that relies on histological features, including tumour multifocality, extrathyroidal extension, and lymph node involvement. The negative effect of N1 (ie, neck lymph node metastases) should not be overestimated. Among patients with N1 disease, the size and number of metastatic lymph nodes as well as the presence or absence of extracapsular extension should be taken into account to assess the effect of N1 on the risk of relapse. ${ }^{54-56}$ In the 2015 American Thyroid Association (ATA) guidelines, patients with N1 disease were classified as low, intermediate, or high risk on the basis of the number and size of N1, whereas in the 2009 ATA classification, all patients with neck lymph node metastases were classified as intermediate risk. ${ }^{55,57}$

Potential histological and biological prognostic factors (including mutation status) have been analysed. Desmoplastic stromal reaction is associated with lymph node metastases and histological invasive features such as peritumoral infiltration and vascular invasion. ${ }^{58}$ Furthermore, an infiltrative growth pattern of the primary tumour and tumour-associated fibrosis is significantly associated with locoregional lymph node involvement at diagnosis. ${ }^{59}$ In a clinicopathological study of 60 patients with papillary thyroid microcarcinoma, multivariate analysis showed peritumoural lymphatic vessel density as the only independent variable associated with a higher rate of locoregional neck metastases. ${ }^{60}$

A combination of tumour size and maximum standardised uptake value of the primary tumour on 18F-FDG PET scanning has been shown to predict the likelihood of central neck lymph node metastasis from papillary thyroid microcarcinoma with a sensitivity of $68 \%$ and a specificity of $71 \% .^{61}$ In another study, researchers showed the absence of correlation between 18F-FDG uptake and BRAF mutation in papillary thyroid microcarcinoma. ${ }^{62}$ However, even when multiple clinical factors are combined, the specificity and positive predictive value of these models are too poor to provide useful

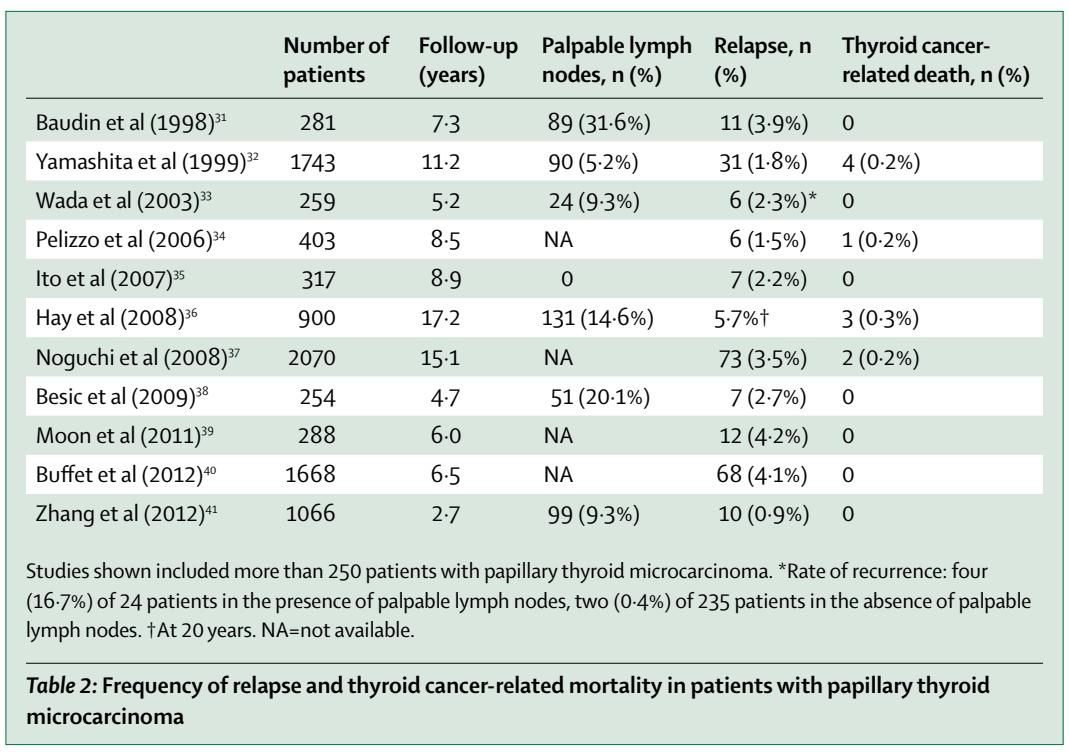

preoperative risk stratification. ${ }^{63}$ Furthermore, many of the potentially important risk factors can only be determined postoperatively on the basis of thyroid pathology.

BRAF mutations are present in $30-67 \%$ of patients with papillary thyroid microcarcinomas ${ }^{6475}$ and are associated with locoregional metastases, extrathyroidal extension, and an increased American Joint Committee on Cancer (AJCC) stage at presentation..$^{67,68,72,74,76,77}$ In a meta-analysis of data from 2247 patients with papillary thyroid microcarcinoma, an increased risk of recurrence was reported in those with a BRAF mutation (odds ratio 2.09 [95\% CI 1.31-3.33]; $\mathrm{p}=0.002$ ). ${ }^{78}$ The histopathological and molecular profiles (BRAF, RAS) of papillary thyroid microcarcinoma were studied in a cohort of 29 patients with locoregional metastases and compared with a cohort of 30 patients matched for age, sex, and tumour size, but with papillary thyroid microcarcinoma confined to the thyroid..$^{46}$ Tumours with locoregional metastases were more likely to have extrathyroidal extension, infiltrative borders, multiple foci, and to harbour a BRAF mutation. Additionally, a subcapsular location of the tumour was also associated with nodal metastases. A predictive score that incorporated three histological features (superficial tumour location, intraglandular spread or multifocality, and tumour fibrosis) with $B R A F$ status was more predictive of locoregional spread than $B R A F$ status or the histological characteristics alone. However, this score has limited applicability to the selection of patients for active surveillance, since two of the variables (multifocality and tumour fibrosis) can only be assessed postoperatively.

TERT promoter mutations are present in fewer than $10 \%$ of patients with papillary thyroid microcarcinoma, ${ }^{79,80}$ with some studies showing a correlation between TERT promoter mutation and aggressive clinicohistological features. Recently, the association of BRAF and TERT 
promoter mutations present in 22 (3\%) of 653 patients with papillary thyroid cancers was associated with clinicopathological features of high-risk differentiated thyroid cancer, including older age, larger tumour size, extrathyroidal invasion, capsular invasion, and advanced disease stages. ${ }^{80}$

The presence of multiple mutations within the same papillary thyroid microcarcinoma (BRAF and TERT or multiple mutations) is rare and might predict a more aggressive disease ${ }^{80,81}$ However, this hypothesis needs to be confirmed in prospective clinical studies. A more comprehensive, integrated assessment of the genome by next-generation sequencing might identify a gene signature in aggressive papillary thyroid microcarcinoma.

\section{Indications for fine needle aspiration}

Thyroid nodules smaller than $1 \mathrm{~cm}$ in diameter that are highly suspected to be papillary thyroid microcarcinomas on the basis of neck ultrasonography that should be submitted to fine needle aspiration biopsy are those for which, if malignancy is confirmed, a resection will be preferred to active surveillance because of ultrasonographic features suggesting a high risk of recurrence-ie, thyroid nodules with the presence of cervical lymph node metastases, extrathyroidal extension, or multiple foci. Thyroid incidentalomas detected by 18F-FDG PET that are smaller than $1 \mathrm{~cm}$ in diameter and have ultrasound criteria of malignancy could also benefit from fine needle aspiration biopsy in rare cases in which the diagnosis of intrathyroid metastases affects the treatment of the other primary cancer. Additionally, suspicious subcentimetre nodules in subcapsular locations where even minor disease progression could be associated with complications (eg, adjacent to recurrent laryngeal nerve or trachea) should also be considered for biopsy. ${ }^{82}$ The exclusion of patients with these nodules from active surveillance is mainly based on the absence of data, because these patients were not offered surveillance in the study by Ito and colleagues..$^{82}$ If active surveillance is chosen for these nodules, it should be done within the frame of prospective trials, since treatment de-escalation should be a prudent process. There is, however, a concern about the level and availability of expertise and experience needed to accurately recognise the presence of extrathyroid extension, multifocality, and at-risk nodules on neck ultrasonography because of their location. Otherwise, thyroid nodules smaller than $1 \mathrm{~cm}$ in size should not be submitted to fine needle aspiration biopsy. This recommendation is unanimous, and is considered a strong recommendation with moderate quality of evidence in the 2015 ATA guidelines. ${ }^{55,83}$

\section{Initial treatment}

\section{Surgery}

In patients with clinical lymph node metastases (cN1), surgery is the standard treatment. In most cases the clinical lymph nodes are located in the lateral compartment. A total thyroidectomy with therapeutic central and lateral lymph node dissection is then recommended because lateral neck metastases are present in $83 \%$ of patients with papillary thyroid microcarcinoma, central neck metastases in $96 \%$, and skip metastases (lateral neck metastases without central neck metastases) in only $4 \% .{ }^{33,55}$ Because the effects of neck dissection on survival have not been clearly shown and because of the morbidity of surgery, even in high-volume centres, the extent of prophylactic central neck dissections done in conjunction with a therapeutic lateral neck dissection remains controversial. Some centres will recommend a bilateral prophylactic central neck dissection, others a unilateral, and less frequently no central prophylactic neck dissection at all. The same uncertainties exist regarding the prophylactic contralateral neck dissection.

In the absence of clinical lymph node involvement (cN0), the low risk of recurrence of papillary thyroid microcarcinoma has to be taken into account and balanced against the risk of permanent complications of total thyroidectomy and the possibility of salvage therapy in case of recurrence. The risk of recurrence is similar after lobectomy and total thyroidectomy in patients with unifocal papillary thyroid microcarcinoma without extrathyroid extension and cN0. Permanent complications of thyroid surgery include permanent hypoparathyroidism and recurrent nerve palsy and can be decreased if surgery is done by highly experienced surgeons. ${ }^{84,85}$ However, even for operations done by experienced surgeons, the risk of permanent hypoparathyroidism and permanent recurrent nerve palsy is $1-3 \%$ following total thyroidectomy and prophylactic neck dissection, higher than following a total thyroidectomy alone or a lobectomy, for which it is less than $1 \%$. The main advantage of a lobectomy is that it carries no risk of permanent hypocalcaemia, by contrast with a total thyroidectomy. ${ }^{86}$

In view of these data, thyroid lobectomy alone is a sufficient treatment for unifocal, intrathyroidal, and cN0 papillary thyroid microcarcinoma in the absence of previous head and neck radiation, familial thyroid carcinoma, and abnormalities of the contralateral thyroid lobe..$^{55}$ Furthermore, following lobectomy, not all patients will need levothyroxine treatment since overt hypothyroidism will occur in only $22 \%$ of cases.$^{87}$ Finally, quality of life is preserved in most, but not all, patients receiving levothyroxine treatment. ${ }^{88}$ The drawback is that follow-up of patients might seem more convenient after a total thyroidectomy compared with a lobectomy. However, recent data show that serum thyroglobulin concentrations can be easily used to assess patient response after a lobectomy. ${ }^{89}$

\section{Radioactive iodine}

The question of whether to use radioactive iodine ablation following a diagnosis of papillary thyroid microcarcinoma only concerns patients who undergo total thyroidectomy. In patients with unifocal papillary 
thyroid microcarcinoma and in the absence of lymph node metastases, there is no indication for postoperative remnant ablation as recommended by the 2009 and 2015 ATA guidelines and by the 2006 European guidelines..$^{55,57,90}$ In patients with multifocal tumours, a risk factor for recurrence, the ATA guidelines do not recommend routine radioactive iodine ablation. Finally, in patients with extrathyroid extension of papillary thyroid microcarcinoma-another risk factor for recurrencethe role of radioactive iodine ablation is controversial, and the benefit of this treatment on recurrence-free survival has not been shown.

\section{Follow-up}

The frequency of follow-up of patients with papillary thyroid microcarcinoma has to be adapted to the indolent course of the disease and to its low level of recurrence. Recurrence can be diagnosed by measurement of thyroglobulin concentration or neck ultrasonography. Following total thyroidectomy without radioactive iodine administration, serum thyroglobulin concentrations are dependent on the amount of thyroid residue left by the surgeon. In expert hands, serum thyroglobulin concentration is expected to be lower than $1 \mathrm{ng} / \mathrm{mL}$ in $96 \%$ of patients and lower than $2 \mathrm{ng} / \mathrm{mL}$ in all patients after total thyroidectomy. ${ }^{91,92}$ Thyroglobulin antibodies, initially present in $10-20 \%$ of patients, are expected to decrease spontaneously. ${ }^{92}$ Following lobectomy, thyroglobulin concentration, and trends in thyroglobulin concentrations and thyroglobulin antibodies over time, can be taken into account to define an excellent response, with a value of less than $30 \mathrm{ng} / \mathrm{mL}$ as a cutoff for thyroglobulin concentration. ${ }^{89}$

The remaining question is the frequency at which these examinations should be done. Unfortunately, prospective clinical data are not available. Thyroglobulin measurement and neck ultrasonography are done at 6-12 months after initial treatment. In the absence of abnormality, serum thyroglobulin measurement every $2-4$ years seems reasonable, and in patients treated with lobectomy a neck ultrasonography every 4-5 years seems reasonable. Because of the absence of evidence, this follow-up strategy of surveillance is a proposition and can only be debated until further convincing data become available.

\section{Active surveillance: an alternative to surgery}

Active surveillance is a strategy initially used in the setting of low-risk localised prostate cancer. It is an approach with a curative intent in which active treatment is delayed until the cancer shows significant progression to avoid (or at least delay) side-effects of treatment. ${ }^{93}$ This strategy can only be used in patients with indolent malignancies. Active surveillance is a new management strategy in oncology for both physicians and patients. As a result, both need to be made aware of and educated about the approach, and physicians need to gain experience of it, especially since patients are heavily influenced by physicians in their decision of whether or
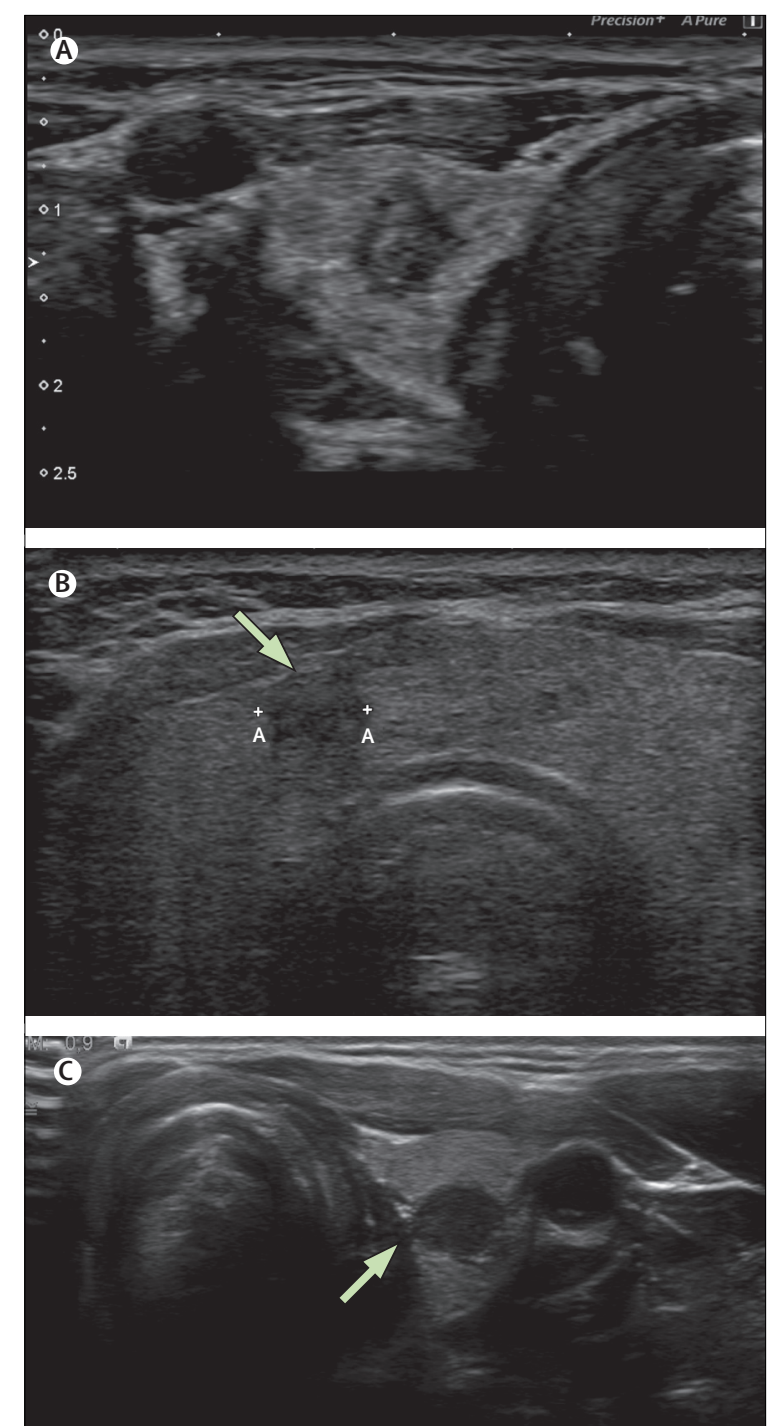

Figure 1: Ultrasound images of patients with papillary thyroid microcarcinoma showing suitability for active surveillance

(A) Suitable for active surveillance because the papillary thyroid microcarcinoma is confined to the thyroid parenchyma. (B) Not suitable for active surveillance because of location close to the thyroid capsule (arrow); the limits of the thyroid microcarcinoma are shown by the two As. (C) Not suitable for active surveillance because of location close to the trachea (arrow is pointing to the thyroid capsule close to the trachea).

not to opt for active surveillance. ${ }^{94}$ Furthermore, the effects of anxiety created by the diagnosis of cancer without receiving active treatment need to be assessed and investigated, and might change with time. ${ }^{95}$ Although the general concept that very low-risk thyroid cancer can be safely observed without immediate treatment is slowly being accepted by patients and physicians, much more experience will be required before this approach will be widely applied in routine clinical practice.

Patients with papillary thyroid microcarcinoma who meet the following criteria are good candidates for active 
surveillance: tumour size of $1 \mathrm{~cm}$ or less, confined to the thyroid parenchyma, not adjacent to the thyroid capsule, or in the absence of abnormal cervical lymph nodes or recurrent nerve palsy (figure 1)..9697 Many factors can affect the decision for or against active surveillance. ${ }^{96}$ These factors include patient characteristics such as age, family history of thyroid cancer, compliance with followup, and willingness to defer immediate surgery (figure 2); and characteristics of the multidisciplinary team such as the experience of the clinician treating the thyroid cancer and the quality of neck ultrasonography. ${ }^{96}$ However, the level of clinician experience is difficult to assess because criteria to define relevant expertise are not available.

So far, all studies of active surveillance in patients with papillary thyroid microcarcinoma have been done in Japan. $43,82,97,98,100$ The active surveillance programme consisted of serial neck ultrasonography every 6-12 months. Significant progression of the primary tumour was defined as an increase in size of $3 \mathrm{~mm}$ or more, or identification of locoregional or distant metastases. The first report on a cohort of 340 patients managed with active surveillance showed that $22(6 \%)$ patients with papillary thyroid microcarcinoma had an increase in tumour size $(\geq 3 \mathrm{~mm})$ at 5 years and $54(16 \%)$ had an increase at 10 years. ${ }^{82}$ Only seven $(1 \%)$ patients developed locoregional lymph node metastases. ${ }^{82}$ No patients developed distant metastases, and no disease-specific deaths were reported. ${ }^{82}$ The update of this prospective cohort study included 1235 patients with papillary thyroid microcarcinoma followed up for more

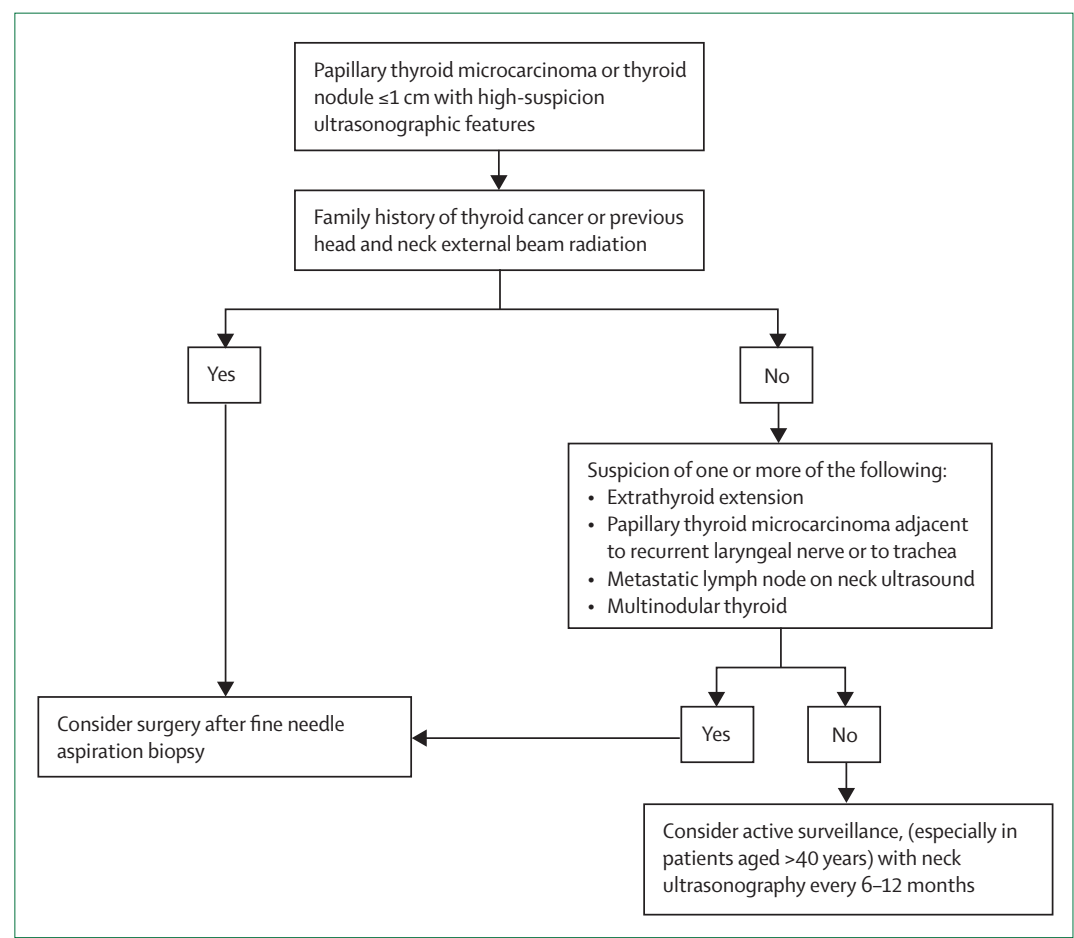

Figure 2: Decision tree for treatment of patients with papillary thyroid microcarcinoma than 1.5 years (mean follow-up 5 years). ${ }^{100}$ Consistent with the initial findings, $58(5 \%)$ patients showed an increase in tumour size during follow-up (ie, an increase of $\geq 3 \mathrm{~mm}$ or a tumour size of $\geq 12 \mathrm{~mm}), 19(2 \%)$ had locoregional lymph node metastases (mostly located in the lateral compartment), and no patients developed distant metastases (table 3)..$^{100}$ All patients who underwent surgery at the time of progression had a favourable outcome, similar to the outcome seen in patients who had immediate surgery. Furthermore, patients undergoing active surveillance had a lower frequency of unfavourable events such as permanent hypoparathyroidism than those who had immediate surgery $(0 \cdot 8 \%$ vs $1 \cdot 6 \%$, respectively; $\mathrm{p}=0 \cdot 0001) .{ }^{43}$ Finally, no thyroid cancer-related deaths were reported in patients under active surveillance or in patients treated with surgery during the same time period at the same institution.

Age is the only identified risk factor for disease progression, with younger patients (aged $<40$ years) being more likely to progress than older patients (table 3). ${ }^{100}$ Tumour size, multiple foci, sex, thyroidstimulating hormone concentration, and family history were not significant prognostic factors for progression. ${ }^{100}$ Disease progression was noted in four of nine patients with papillary thyroid microcarcinoma under observation during pregnancy. ${ }^{101}$ However, an updated assessment showed disease progression in only four $(8 \%)$ of 50 patients with papillary thyroid microcarcinoma examined before and after a full-term pregnancy. ${ }^{102}$ Findings from a recent study suggested that changes in ultrasonographic pattern during follow-up might predict progression, with consolidation of calcification and loss of vascularity during follow-up being significant indicators for non-progressive disease..$^{103}$

These data suggest that a long-term active surveillance management approach might be more effective and appropriate in older patients than in younger patients. However, younger patients could also opt for an active surveillance approach for deferral of surgical intervention to a more convenient time. The drawback of active surveillance is its acceptance by patients. Indeed, half of patients from the centre that initiated the approach in thyroid cancer and promoted it through a prospective trial chose immediate surgery rather than surveillance. ${ }^{43}$

Other than age, there is no identified predictor of progression of papillary thyroid microcarcinoma during active surveillance. Whether the molecular profiling of papillary thyroid microcarcinoma can be useful to decide whether active surveillance can be offered is a question that needs to be answered. A large genomic analysis might be more pertinent than an analysis focusing on $B R A F$ and TERT mutations. In view of the indolent natural history of papillary thyroid microcarcinomas, the question of the cost-effectiveness of active surveillance will also have to be addressed. 


\begin{tabular}{|c|c|c|c|c|c|c|}
\hline & \multicolumn{2}{|c|}{ Increase in tumour size by $3 \mathrm{~mm}$ or more } & \multicolumn{2}{|c|}{ Appearance of lymph node metastases } & \multicolumn{2}{|c|}{ Progression to clinical disease ${ }^{*}$} \\
\hline & 5 years & 10 years & 5 years & 10 years & 5 years & 10 years \\
\hline$<40$ years & 45 (9.1\%) & 11 (12.1\%) & $49(5 \cdot 2 \%)$ & $13(16 \cdot 1 \%)$ & $48(9.5 \%)$ & $13(22.5 \%)$ \\
\hline 40-59years & $225(5 \cdot 0 \%)$ & 82 (9.1\%) & $229(1.4 \%)$ & $87(2 \cdot 3 \%)$ & $229(4 \cdot 0 \%)$ & $87(4 \cdot 9 \%)$ \\
\hline$\geq 60$ years & $157(4.0 \%)$ & $36(4 \cdot 0 \%)$ & $160(0.5 \%)$ & $36(0.5 \%)$ & $157(2 \cdot 2 \%)$ & $36(2 \cdot 5 \%)$ \\
\hline
\end{tabular}

All data for active surveillance have been obtained in Japan and confirmation by prospective trials in North America and Europe are needed. However, on the basis of these data and a careful consideration of the risks versus benefits of immediate surgical intervention in papillary thyroid microcarcinoma, the authors of the 2015 ATA guidelines note that, although surgery is generally recommended for cytologically confirmed thyroid cancer, an active surveillance management approach "can be considered" as an alternative to immediate surgery in patients with very low-risk tumours, such as papillary microcarcinomas without other known high-risk clinical, cytological, or molecular (if assessed) features. ${ }^{55}$ An effective active surveillance management plan requires careful patient selection, meticulous neck ultrasound follow-up, and a shared treatment philosophy between the patient and all members of the disease management team. The optimum patient for active surveillance is an older patient ( $>40$ years) with a unifocal papillary microcarcinoma with no ultrasound evidence of extrathyroidal extension or cervical lymph node involvement in the setting of an otherwise normal thyroid ultrasound, and who is willing to return at 6-12-month intervals for serial ultrasound examinations over many years.

\section{Local destruction: an alternative to surgery?}

Radiofrequency ablation, ethanol injection, and laser ablation were first developed for the treatment of distant metastases from thyroid cancer, locoregional recurrences of thyroid cancer, and benign nodules. ${ }^{104,105}$ Treatment of papillary thyroid microcarcinomas with these techniques has been little studied. Recent reports of needle track tumour seeding after radiofrequency ablation of a thyroid cancer and incomplete destruction of a thyroid cancer should lead to a prospective assessment of these techniques. ${ }^{106,107}$ A feasibility study in three patients treated with laser ablation followed by surgery was recently reported. ${ }^{108}$ The primary papillary thyroid microcarcinomas were destroyed, leaving the problems of multifocality and perithyroid metastatic lymph nodes. These results emphasise the question of whether papillary thyroid microcarcinomas need treatment or not.

\section{Conclusions}

To address recent concerns regarding overdiagnosis and overtreatment of papillary thyroid microcarcinomas, an

\section{Search strategy and selection criteria}

We searched PubMed with the keywords "papillary thyroid microcarcinoma", "active surveillance", and "thyroid cancer incidence" for articles published between Jan 1, 1980, and June 28,2016 . We focused on those articles published after 2005 and in English because most of the studies in peerreviewed indexed journals are published in English. We also included relevant studies cited in reports identified by this search strategy.

active surveillance approach in which active treatment is delayed until the cancer shows signs of substantial progression should be considered in selected patients. Criteria for selection include the absence of clinical lymph node metastases, absence of ultrasound signs of extrathyroid extension, and location of the nodule distant from the recurrent laryngeal nerve or trachea. Available data suggest the safety of active surveillance, but further prospective data from the USA and Europe are needed. The drawbacks of active surveillance are its poor acceptance by patients and the need for frequent followup. A renaming of the term papillary thyroid microcarcinoma to indolent lesion of epithelial origin will not solve all problems because we will still need to identify the few lesions that will progress and need surgery. In this context, genomic analysis should be investigated for its ability to determine which patients with papillary thyroid microcarcinoma require surgery and those who do not need follow-up.

Contributors

All authors contributed to the literature search, figures, and writing of the Review, and all approved the final submitted and revised versions.

Declaration of interests

We declare no competing interests.

References

1 Sampson RJ, Woolner LB, Bahn RC, Kurland LT. Occult thyroid carcinoma in Olmsted County, Minnesota: prevalence at autopsy compared with that in Hiroshima and Nagasaki, Japan. Cancer 1974 34: 2072-76.

2 Sobrinho-Simoes MA, Sambade MC, Goncalves V. Latent thyroid carcinoma at autopsy: a study from Oporto, Portugal. Cancer 1979; 43: 1702-06.

3 Bondeson L, Ljungberg O. Occult thyroid carcinoma at autopsy in Malmo, Sweden. Cancer 1981; 47: 319-23.

4 Harach HR, Franssila KO, Wasenius VM. Occult papillary carcinoma of the thyroid. A "normal" finding in Finland. A systematic autopsy study. Cancer 1985; 56: 531-38. 
5 Lang W, Borrusch H, Bauer L. Occult carcinomas of the thyroid. Evaluation of 1,020 sequential autopsies. Am J Clin Pathol 1988; 90: 72-76.

6 Ottino A, Pianzola HM, Castelletto RH. Occult papillary thyroid carcinoma at autopsy in La Plata, Argentina. Cancer 1989; 64: 547-51.

7 Yamamoto Y, Maeda T, Izumi K, Otsuka H. Occult papillary carcinoma of the thyroid. A study of 408 autopsy cases. Cancer 1990 65: 1173-79.

8 Furmanchuk AW, Roussak N, Ruchti C. Occult thyroid carcinomas in the region of Minsk, Belarus. An autopsy study of 215 patients. Histopathology 1993; 23: 319-25.

9 Martinez-Tello FJ, Martinez-Cabruja R, Fernandez-Martin J, Lasso-Oria C, Ballestin-Carcavilla C. Occult carcinoma of the thyroid. A systematic autopsy study from Spain of two series performed with two different methods. Cancer 1993; 71: 4022-29.

10 Kovacs GL, Gonda G, Vadasz G, et al. Epidemiology of thyroid microcarcinoma found in autopsy series conducted in areas of different iodine intake. Thyroid 2005; 15: 152-57.

11 Neuhold N, Kaiser H, Kaserer K. Latent carcinoma of the thyroid in Austria: a systematic autopsy study. Endocr Pathol 2001; 12: 23-31.

12 Lee YS, Lim H, Chang HS, Park CS. Papillary thyroid microcarcinomas are different from latent papillary thyroid carcinomas at autopsy. J Korean Med Sci 2014; 29: 676-79.

13 Leenhardt L, Grosclaude P, Cherie-Challine L. Increased incidence of thyroid carcinoma in France: a true epidemic or thyroid nodule management effects? Report from the French Thyroid Cancer Committee. Thyroid 2004; 14: 1056-60.

14 McLeod DS, Sawka AM, Cooper DS. Controversies in primary treatment of low-risk papillary thyroid cancer. Lancet 2013; 381: 1046-57.

15 Enewold LR, Zhou J, Devesa SS, et al. Thyroid cancer incidence among active duty US military personnel, 1990-2004. Cancer Epidemiol Biomarkers Prev 2011; 20: 2369-76.

16 Rego-Iraeta A, Perez-Mendez LF, Mantinan B, Garcia-Mayor RV. Time trends for thyroid cancer in northwestern Spain: true rise in the incidence of micro and larger forms of papillary thyroid carcinoma. Thyroid 2009; 19: 333-40.

17 Davies L, Welch HG. Increasing incidence of thyroid cancer in the United States, 1973-2002. JAMA 2006; 295: 2164-67.

18 Hughes DT, Haymart MR, Miller BS, Gauger PG, Doherty GM. The most commonly occurring papillary thyroid cancer in the United States is now a microcarcinoma in a patient older than 45 years. Thyroid 2011; 21 : 231-36.

19 Altekruse S, Das A, Cho H, Petkov V, Yu M. Do US thyroid cancer incidence rates increase with socioeconomic status among people with health insurance? An observational study using SEER population-based data. BMJ Open 2015; 5: e009843.

20 Brito JP, Al Nofal A, Montori VM, Hay ID, Morris JC. The impact of subclinical disease and mechanism of detection on the rise in thyroid cancer incidence: a population-based study in Olmsted County, Minnesota during 1935 through 2012. Thyroid 2015; 25: 999-1007.

21 Vaccarella S, Dal Maso L, Laversanne M, Bray F, Plummer M, Franceschi S. The impact of diagnostic changes on the rise in thyroid cancer incidence: a population-based study in selected high-resource countries. Thyroid 2015; 25: 1127-36.

22 Ahn HS, Kim HJ, Welch HG. Korea's thyroid-cancer "epidemic"screening and overdiagnosis. N Engl J Med 2014; 371: 1765-67.

23 Ahn HS, Welch HG. South Korea's thyroid-cancer "epidemic" turning the tide. N Engl J Med 2015; 373: 2389-90.

24 Tsuda T, Tokinobu A, Yamamoto E, Suzuki E. Thyroid cancer detection by ultrasound among residents ages 18 years and younger in Fukushima, Japan: 2011 to 2014. Epidemiology 2016; 27: 316-22.

25 Morris LG, Sikora AG, Tosteson TD, Davies L. The increasing incidence of thyroid cancer: the influence of access to care. Thyroid 2013; 23: 885-91

26 Sassolas G, Hafdi-Nejiari Z, Remontet L, et al. Thyroid cancer: is the incidence rise abating? Eur J Endocrinol 2009; 160: 71-79.

27 Busco S, Buzzoni C, Mallone S, et al. Italian cancer figures-report 2015: the burden of rare cancers in Italy. Epidemiol Prev 2016; 40 (1 suppl 2): 1-120.
28 Esserman LJ, Thompson IM, Reid B, et al. Addressing overdiagnosis and overtreatment in cancer: a prescription for change. Lancet Oncol 2014; 15: e234-42.

29 Cronan JJ. Thyroid nodules: is it time to turn off the US machines? Radiology 2008; 247: 602-04.

30 Howlett DC, Speirs A. The thyroid incidentaloma-ignore or investigate? J Ultrasound Med 2007; 26: 1367-71.

31 Baudin E, Travagli JP, Ropers J, et al. Microcarcinoma of the thyroid gland: the Gustave-Roussy Institute experience. Cancer 1998; 83: 553-59.

32 Yamashita H, Noguchi S, Murakami N, et al. Extracapsular invasion of lymph node metastasis. A good indicator of disease recurrence and poor prognosis in patients with thyroid microcarcinoma. Cancer 1999; 86: 842-49.

33 Wada N, Duh QY, Sugino K, et al. Lymph node metastasis from 259 papillary thyroid microcarcinomas: frequency, pattern of occurrence and recurrence, and optimal strategy for neck dissection. Ann Surg 2003; 237: 399-407.

34 Pelizzo MR, Boschin IM, Toniato A, et al. Papillary thyroid microcarcinoma (PTMC): prognostic factors, management and outcome in 403 patients. Eur J Surg Oncol 2006; 32: 1144-48.

35 Ito Y, Higashiyama T, Takamura Y, et al. Prognosis of patients with benign thyroid diseases accompanied by incidental papillary carcinoma undetectable on preoperative imaging tests. World J Surg 2007; 31: 1672-76.

36 Hay ID, Hutchinson ME, Gonzalez-Losada T, et al. Papillary thyroid microcarcinoma: a study of 900 cases observed in a 60-year period. Surgery 2008; 144: 980-87.

37 Noguchi S, Yamashita H, Uchino S, Watanabe S. Papillary microcarcinoma. World J Surg 2008; 32: 747-53.

38 Besic N, Zgajnar J, Hocevar M, Petric R. Extent of thyroidectomy and lymphadenectomy in 254 patients with papillary thyroid microcarcinoma: a single-institution experience. Ann Surg Oncol 2009; 16: 920-28.

39 Moon HJ, Kim EK, Chung WY, Yoon JH, Kwak JY. Minimal extrathyroidal extension in patients with papillary thyroid microcarcinoma: is it a real prognostic factor? Ann Surg Oncol 2011; 18: 1916-23.

40 Buffet C, Golmard JL, Hoang C, et al. Scoring system for predicting recurrences in patients with papillary thyroid microcarcinoma. Eur J Endocrinol 2012; 167: 267-75.

41 Zhang L, Wei WJ, Ji QH, et al. Risk factors for neck nodal metastasis in papillary thyroid microcarcinoma: a study of 1066 patients. J Clin Endocrinol Metab 2012; 97: 1250-57.

42 Mehanna H, Al-Maqbili T, Carter B, et al. Differences in the recurrence and mortality outcomes rates of incidental and nonincidental papillary thyroid microcarcinoma: a systematic review and meta-analysis of 21329 person-years of follow-up. J Clin Endocrinol Metab 2014; 99: 2834-43.

43 Miyauchi A. Clinical trials of active surveillance of papillary microcarcinoma of the thyroid. World J Surg 2016; 40: 516-22.

44 Chow SM, Law SC, Chan JK, Au SK, Yau S, Lau WH. Papillary microcarcinoma of the thyroid--prognostic significance of lymph node metastasis and multifocality. Cancer 2003; 98: 31-40.

45 Giordano D, Gradoni P, Oretti G, Molina E, Ferri T. Treatment and prognostic factors of papillary thyroid microcarcinoma. Clin Otolaryngol 2010; 35: 118-24.

46 Niemeier LA, Kuffner Akatsu H, Song C, et al. A combined molecular-pathologic score improves risk stratification of thyroid papillary microcarcinoma. Cancer 2012; 118: 2069-77.

47 Ito Y, Tomoda C, Uruno T, et al. Ultrasonographically and anatomopathologically detectable node metastases in the latera compartment as indicators of worse relapse-free survival in patients with papillary thyroid carcinoma. World J Surg 2005; 29: 917-20.

48 Roh JL, Kim JM, Park CI. Central cervical nodal metastasis from papillary thyroid microcarcinoma: pattern and factors predictive of nodal metastasis. Ann Surg Oncol 2008; 15: 2482-86.

49 Zhao Q, Ming J, Liu C, et al. Multifocality and total tumor diameter predict central neck lymph node metastases in papillary thyroid microcarcinoma. Ann Surg Oncol 2013; 20: 746-52.

50 Kim KE, Kim EK, Yoon JH, Han KH, Moon HJ, Kwak JY. Preoperative prediction of central lymph node metastasis in thyroid papillary microcarcinoma using clinicopathologic and sonographic features. World J Surg 2013; 37: 385-91. 
51 Ardito G, Revelli L, Giustozzi E, et al. Aggressive papillary thyroid microcarcinoma: prognostic factors and therapeutic strategy. Clin Nucl Med 2013; 38: 25-28.

52 Cho JK, Kim JY, Jeong CY, et al. Clinical features and prognostic factors in papillary thyroid microcarcinoma depends on age. J Korean Surg Soc 2012; 82: 281-87.

53 Roti E, degli Uberti EC, Bondanelli M, Braverman LE. Thyroid papillary microcarcinoma: a descriptive and meta-analysis study. Eur J Endocrinol 2008; 159: 659-73.

54 Leboulleux S, Rubino C, Baudin E, et al. Prognostic factors for persistent or recurrent disease of papillary thyroid carcinoma with neck lymph node metastases and/or tumor extension beyond the thyroid capsule at initial diagnosis. J Clin Endocrinol Metab 2005; 90: 5723-29.

55 Haugen BR, Alexander EK, Bible KC, et al. 2015 American Thyroid Association management guidelines for adult patients with thyroid nodules and differentiated thyroid cancer: The American Thyroid Association Guidelines Task Force on Thyroid Nodules and Differentiated Thyroid Cancer. Thyroid 2016; 26: 1-133.

56 Randolph GW, Duh QY, Heller KS, et al. The prognostic significance of nodal metastases from papillary thyroid carcinoma can be stratified based on the size and number of metastatic lymph nodes, as well as the presence of extranodal extension. Thyroid 2012 22: $1144-52$.

57 Cooper DS, Doherty GM, Haugen BR, et al. Revised American Thyroid Association management guidelines for patients with thyroid nodules and differentiated thyroid cancer. Thyroid 2009; 19: 1167-214.

58 Koperek O, Asari R, Niederle B, Kaserer K. Desmoplastic stromal reaction in papillary thyroid microcarcinoma. Histopathology 2011; 58: 919-24.

59 Chung YJ, Lee JS, Park SY, et al. Histomorphological factors in the risk prediction of lymph node metastasis in papillary thyroid carcinoma. Histopathology 2013; 62: 578-88.

60 Chung MK, Kim JH, Ko YH, Son YI. Correlation of lymphatic vessel density and vascular endothelial growth factor with nodal metastasis in papillary thyroid microcarcinoma. Head Neck 2012; 34: 846-51.

61 Byun BH, Jeong UG, Hong SP, et al. Prediction of central lymph node metastasis from papillary thyroid microcarcinoma by ${ }^{18}$ F-fluorodeoxyglucose PET/CT and ultrasonography. Ann Nucl Med 2012; 26: 471-77.

62 Yoon S, An YS, Lee SJ, et al. Relation between F-18 FDG uptake of PET/CT and BRAFV600E mutation in papillary thyroid cancer. Medicine (Baltimore) 2015; 94: e2063.

63 Yu XM, Wan Y, Sippel RS, Chen H. Should all papillary thyroid microcarcinomas be aggressively treated? An analysis of 18,445 cases. Ann Surg 2011; 254: 653-60.

64 Kimura ET, Nikiforova MN, Zhu Z, Knauf JA, Nikiforov YE, Fagin JA. High prevalence of $B R A F$ mutations in thyroid cancer: genetic evidence for constitutive activation of the RET/PTC-RASBRAF signaling pathway in papillary thyroid carcinoma. Cancer Res 2003; 63: 1454-57.

65 Nikiforova MN, Kimura ET, Gandhi M, et al. BRAF mutations in thyroid tumors are restricted to papillary carcinomas and anaplastic or poorly differentiated carcinomas arising from papillary carcinomas. J Clin Endocrinol Metab 2003; 88: 5399-404.

66 Xing M, Westra WH, Tufano RP, et al. BRAF mutation predicts a poorer clinical prognosis for papillary thyroid cancer. $J$ Clin Endocrinol Metab 2005; 90: 6373-79.

67 Kim TY, Kim WB, Rhee YS, et al. The BRAF mutation is useful for prediction of clinical recurrence in low-risk patients with conventional papillary thyroid carcinoma. Clin Endocrinol (Oxf) 2006; 65: 364-68.

68 Kwak JY, Kim EK, Chung WY, Moon HJ, Kim MJ, Choi JR. Association of BRAFV600E mutation with poor clinical prognostic factors and US features in Korean patients with papillary thyroid microcarcinoma. Radiology 2009; 253: 854-60.

69 Xing M. BRAF mutation in papillary thyroid microcarcinoma: the promise of better risk management. Ann Surg Oncol 2009; 16: 801-03.

70 Lin KL, Wang OC, Zhang XH, Dai XX, Hu XQ, Qu JM. The BRAF mutation is predictive of aggressive clinicopathological characteristics in papillary thyroid microcarcinoma. Ann Surg Oncol 2010; 17: 3294-300.
71 Park YJ, Kim YA, Lee YJ, et al. Papillary microcarcinoma in comparison with larger papillary thyroid carcinoma in $B R A F^{\mathrm{V} 600 \mathrm{E}}$ mutation, clinicopathological features, and immunohistochemical findings. Head Neck 2010; 32: 38-45.

72 Zheng X, Wei S, Han Y, et al. Papillary microcarcinoma of the thyroid: clinical characteristics and BRAF ${ }^{\mathrm{V} 600 \mathrm{E}}$ mutational status of 977 cases. Ann Surg Oncol 2013; 20: 2266-73.

73 Bastos AU, Oler G, Nozima BH, Moyses RA, Cerutti JM. $B R A F$ V600E and decreased NIS and TPO expression are associated with aggressiveness of a subgroup of papillary thyroid microcarcinoma. Eur J Endocrinol 2015; 173: 525-40.

74 Li F, Chen G, Sheng C, et al. BRAFV600E mutation in papillary thyroid microcarcinoma: a meta-analysis. Endocr Relat Cancer 2015; 22: $159-68$

75 Tallini G, de Biase D, Durante C, et al. BRAF V600E and risk stratification of thyroid microcarcinoma: a multicenter pathological and clinical study. Mod Pathol 2015; 28: 1343-59.

76 Lee X, Gao M, Ji Y, et al. Analysis of differential BRAF $F^{\mathrm{v} 600}$ mutational status in high aggressive papillary thyroid microcarcinoma. Ann Surg Oncol 2009; 16: 240-45.

77 Rossi ED, Martini M, Capodimonti S, et al. BRAF (V600E) mutation analysis on liquid-based cytology-processed aspiration biopsies predicts bilaterality and lymph node involvement in papillary thyroid microcarcinoma. Cancer Cytopathol 2013; 121: 291-97.

78 Chen Y, Sadow PM, Suh H, et al. BRAF $F^{\mathrm{v} 600 \mathrm{E}}$ is correlated with recurrence of papillary thyroid microcarcinoma: a systematic review, multi-institutional primary data analysis, and meta-analysis. Thyroid 2016; 26: 248-55.

79 de Biase D, Gandolfi G, Ragazzi M, et al. TERT promoter mutations in papillary thyroid microcarcinomas. Thyroid 2015; 25: 1013-19.

80 Jin L, Chen E, Dong S, et al. BRAF and TERT promoter mutations in the aggressiveness of papillary thyroid carcinoma: a study of 653 patients. Oncotarget 2016; published online March 1. DOI:10.18632/oncotarget.7811.

81 Shrestha RT, Karunamurthy A, Amin K, Nikiforov YE, Caramori ML. Multiple mutations detected preoperatively may predict aggressive behavior of papillary thyroid cancer and guide management-a case report. Thyroid 2015; 25: 1375-78.

82 Ito $\mathrm{Y}$, Miyauchi A, Inoue $\mathrm{H}$, et al. An observational trial for papillary thyroid microcarcinoma in Japanese patients. World J Surg 2010; 34: 28-35.

83 Hoang JK, Langer JE, Middleton WD, et al. Managing incidental thyroid nodules detected on imaging: white paper of the ACR Incidental Thyroid Findings Committee. J Am Coll Radiol 2015; 12: $143-50$

84 Kandil E, Noureldine SI, Abbas A, Tufano RP. The impact of surgical volume on patient outcomes following thyroid surgery. Surgery 2013; 154: 1346-52.

85 Sosa JA, Bowman HM, Tielsch JM, Powe NR, Gordon TA, Udelsman R. The importance of surgeon experience for clinical and economic outcomes from thyroidectomy. Ann Surg 1998; 228: 320-30.

86 Hartl DM, Schlumberger M. Extent of thyroidectomy and incidence of morbidity: risk-appropriate treatment. In: Miccoli P, Terris DI, Minuto MN, Seybt MW, eds. Thyroid surgery: preventing and managing complications. Oxford: Wiley-Blackwell, 2013: 19-32.

87 Verloop H, Louwerens M, Schoones JW, Kievit J, Smit JW, Dekkers OM. Risk of hypothyroidism following hemithyroidectomy: systematic review and meta-analysis of prognostic studies. J Clin Endocrinol Metab 2012; 97: 2243-55.

88 Biondi B, Wartofsky L. Combination treatment with T4 and T3: toward personalized replacement therapy in hypothyroidism? J Clin Endocrinol Metab 2012; 97: 2256-71.

89 Momesso DP, Vaisman F, Yang SP, et al. Dynamic risk stratification in differentiated thyroid cancer patients treated without radioactive iodine. J Clin Endocrinol Metab 2016; 101: 2692-700.

90 Pacini F, Schlumberger M, Dralle H, Elisei R, Smit JW Wiersinga W. European consensus for the management of patients with differentiated thyroid carcinoma of the follicular epithelium. Eur J Endocrinol 2006; 154: 787-803. 
91 Durante C, Montesano T, Attard M, et al. Long-term surveillance of papillary thyroid cancer patients who do not undergo postoperative radioiodine remnant ablation: is there a role for serum thyroglobulin measurement? J Clin Endocrinol Metab 2012; 97: 2748-53.

92 Nascimento C, Borget I, Troalen F, et al. Ultrasensitive serum thyroglobulin measurement is useful for the follow-up of patients treated with total thyroidectomy without radioactive iodine ablation. Eur J Endocrinol 2013; 169: 689-93.

93 Chen RC, Rumble RB, Loblaw DA, et al. Active surveillance for the management of localized prostate cancer (Cancer Care Ontario Guideline): American Society of Clinical Oncology Clinical Practice Guideline Endorsement. J Clin Oncol 2016; 34: 2182-90.

94 Gorin MA, Soloway CT, Eldefrawy A, Soloway MS. Factors that influence patient enrollment in active surveillance for low-risk prostate cancer. Urology 2011; 77: 588-91.

95 Pham KN, Cullen J, Hurwitz LM, et al. Prospective quality of life in men choosing active surveillance compared to those biopsied but not diagnosed with prostate cancer. J Urol 2016; published online March 11. DOI:10.1016/j.juro.2016.02.2972.

96 Brito JP, Ito Y, Miyauchi A, Tuttle RM. A clinical framework to facilitate risk stratification when considering an active surveillance alternative to immediate biopsy and surgery in papillary microcarcinoma. Thyroid 2016; 26: $144-49$.

97 Sugitani I, Toda K, Yamada K, Yamamoto N, Ikenaga M, Fujimoto Y. Three distinctly different kinds of papillary thyroid microcarcinoma should be recognized: our treatment strategies and outcomes. World J Surg 2010; 34: 1222-31.

98 Ito Y, Uruno T, Nakano K, et al. An observation trial without surgical treatment in patients with papillary microcarcinoma of the thyroid. Thyroid 2003; 13: 381-87.

99 Sugitani I, Kasai N, Fujimoto Y, Yanagisawa A. A novel classification system for patients with PTC: addition of the new variables of large ( $3 \mathrm{~cm}$ or greater) nodal metastases and reclassification during the follow-up period. Surgery 2004; 135: 139-48.
100 Ito Y, Miyauchi A, Kihara M, Higashiyama T, Kobayashi K, Miya A. Patient age is significantly related to the progression of papillary microcarcinoma of the thyroid under observation. Thyroid 2014; 24: 27-34.

101 Shindo H, Amino N, Ito Y, et al. Papillary thyroid microcarcinoma might progress during pregnancy. Thyroid 2014; 24: 840-44.

102 Ito Y, Miyauchi A, Kudo T, et al. Effects of pregnancy on papillary microcarcinomas of the thyroid re-evaluated in the entire patient series at Kuma Hospital. Thyroid 2016; 26: 156-60.

103 Fukuoka O, Sugitani I, Ebina A, Toda K, Kawabata K, Yamada K. Natural history of asymptomatic papillary thyroid microcarcinoma: time-dependent changes in calcification and vascularity during active surveillance. World J Surg 2016; 40: 529-37.

104 Na DG, Lee JH, Jung SL, et al. Radiofrequency ablation of benign thyroid nodules and recurrent thyroid cancers: consensus statement and recommendations. Korean J Radiol 2012; 13: 117-25.

105 Lewis BD, Hay ID, Charboneau JW, McIver B, Reading CC, Goellner JR. Percutaneous ethanol injection for treatment of cervical lymph node metastases in patients with papillary thyroid carcinoma. AJR Am J Roentgenol 2002; 178: 699-704.

106 Kim HY, Ryu WS, Woo SU, et al. Primary papillary thyroid carcinoma previously treated incompletely with radiofrequency ablation. J Cancer Res Ther 2010; 6: 310-12.

107 Lee CU, Kim SJ, Sung JY, Park SH, Chong S, Baek JH. Needle track tumor seeding after radiofrequency ablation of a thyroid tumor. Jpn J Radiol 2014; 32: 661-63.

108 Valcavi R, Piana S, Bortolan GS, Lai R, Barbieri V, Negro R. Ultrasound-guided percutaneous laser ablation of papillary thyroid microcarcinoma: a feasibility study on three cases with pathological and immunohistochemical evaluation. Thyroid 2013; 23: 1578-82. 